TITLE:

\title{
Operation policy for micro chemical plants with external numbering-up structure
}

\section{$\operatorname{AUTHOR}(S)$ :}

Tonomura, Osamu; Tominari, Takayuki; Kano, Manabu; Hasebe, Shinji

\section{CITATION:}

Tonomura, Osamu ... [et al]. Operation policy for micro chemical plants with external numbering-up structure. Chemical Engineering Journal 2008, 135(1): S131-S137

\section{ISSUE DATE:}

2008-01-15

URL:

http://hdl.handle.net/2433/85261

\section{RIGHT:}

Copyright (C) 2007 Elsevier B.V.; この論文は出版社版でありません。引 用の際には出版社版をご確認ご利用ください。; This is not the published version. Please cite only the published version. 


\title{
Operation Policy for Micro Chemical Plants with External
}

\section{Numbering-up Structure}

\author{
Osamu Tonomura*, Takayuki Tominari, Manabu Kano, Shinji Hasebe \\ Department of Chemical Engineering, Kyoto University \\ Nishikyo-ku, Kyoto 615-8510, Japan \\ * Corresponding author. Tel.: +81-75-383-2677; fax: +81-75-383-2657. \\ E-mail address: tonomura@cheme.kyoto-u.ac.jp (O. Tonomura)
}

\begin{abstract}
One of the critical operational issues of micro chemical plants with external numberingup structure is to keep a uniform flow distribution among parallelized microdevices even when blockage occurs in one or more microdevice. Since it is not practical to install flow controllers in all the microdevices, a simple and effective operation policy against blockage occurrence needs to be developed. In this research, micro chemical plants having four or eight parallelized microdevices are constructed to analyze the influence of blockage on the flow distribution among the parallelized microdevices. The numerical and experimental results show that pressure drop control is superior to total flow control. In addition, two control structures based on pressure drop control, pumping pressure control and pressure drop control over the parallelized section are investigated. It is clarified that the latter control structure enables us to successfully keep the flow rate in each unblocked microdevice at a normal level when blockage occurs. Pressure drop control over the parallelized section is applied to a micro chemical plant having four parallelized micro heat exchangers, and its validity is demonstrated.
\end{abstract}

Keywords: Micro chemical plants, External numbering-up, Flow distribution, Blockage, Process control 


\section{Introduction}

Micro chemical processes have numerous advantages such as improved reaction conversion and selectivity, enhanced temperature control, and increased safety as a result of smaller volume [1]. Instead of the scale-up, the production capacity of micro chemical plants is usually increased by building an internal and/or external numbering-up structure [2]. Internal numbering-up is the parallelization of some components such as microchannels in one microdevice as shown in Fig. 1, and external numbering-up is the parallel setup of some microdevices as shown in Fig. 2.

The performance of micro chemical plants with numbering-up structure depends on the flow distribution among the parallelized microchannels/microdevices [3]. It is desired to achieve equalization of flow rates of all the microchannels/microdevices because flow maldistribution deteriorates reaction selectivity, heat transfer efficiency, and so on. The flow rate in each microchannel/microdevice can be adjusted by installing a flow controller. However, it is not practical to install flow controllers in all the microchannels/microdevices. Therefore, other simple operation strategies need to be proposed to uniformalize the flow distribution. In addition, in real production microchannels are inevitably blocked or clogged, and such an abnormal condition negatively affects whole micro chemical plants. For example, Thomas et al. [3], who developed a continuous radical polymerization process with a micro premixer, mentioned that bad mixing conditions cause fouling in a reactor. Therefore, the proposed operation strategies must function effectively even when blockage occurs in the parallelized microdevices. 

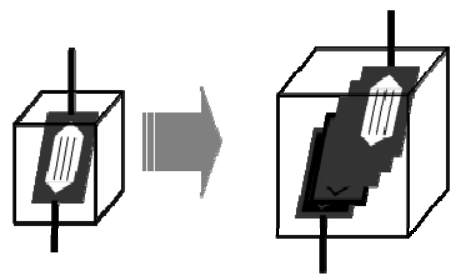

Fig. 1 Internal numbering-up
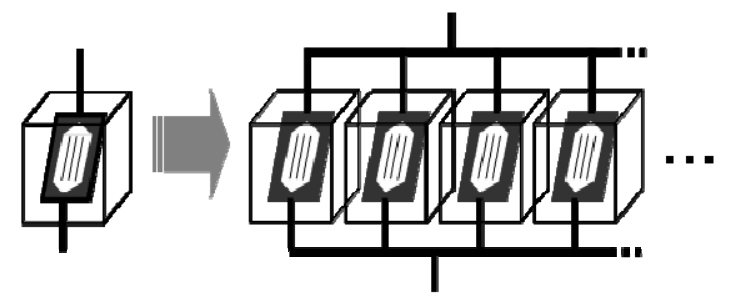

Fig. 2 External numbering-up

In this research, two types of operation policies, total flow control and pressure drop control, are compared from the viewpoint of flow uniformity when blockage occurs. In addition, for pressure drop control, two different control structures, pumping pressure control and pressure drop control over the parallelized section, are investigated through both simulations and experiments. Finally, the proposed control structure is applied to a micro chemical plant composed of four parallelized micro heat exchangers.

\section{Total flow control and pressure drop control}

To maintain the desired product quality, it is important to keep a uniform flow rate in each microdevice of the micro chemical plant when blockage occurs, because flow maldistribution worsens the performance of the micro chemical plant. In this research, pressure drop control is proposed to achieve the uniform flow distribution.

(A)

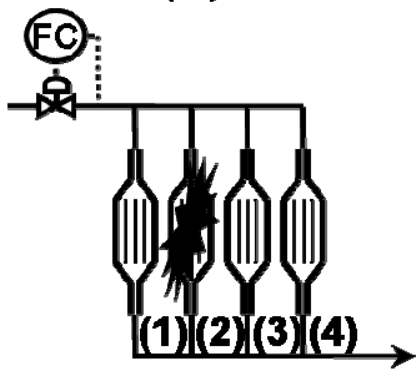

(B)

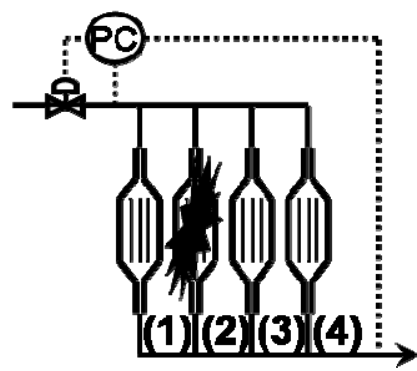

Fig. 3 Parallelized microdevices under two operation policies: (A) total flow control, (B) pressure drop control 
(A)

돈

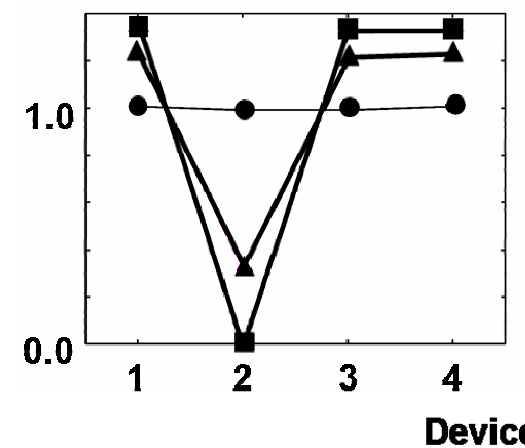

(B)

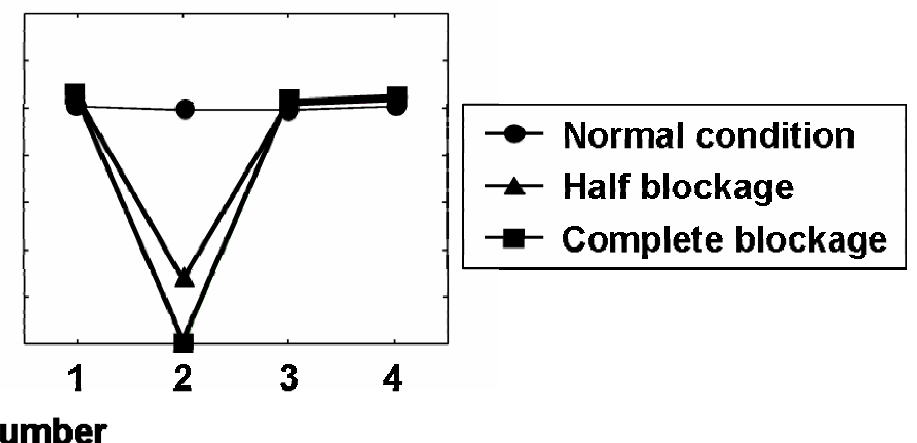

Fig. 4 Mass flow rate of each microdevice under blockage occurrence: (A) total flow control, (B) pressure drop control

A micro chemical plant consisting of four parallelized microdevices in Fig. 3 is used to demonstrate the difference of two operation policies, total flow control and pressure drop control. When blockage occurs in microdevice 2, the flow distribution under the pressure drop control is derived by simulation and compared with that under the total flow control. In Fig. 3, reactant is fed to the parallelized microdevices at $0.1 \mathrm{~m} / \mathrm{s}$ in the normal condition. The physical properties of the reactant are assumed to be the same as water (293 K).

The results are shown in Fig. 4. In the case of blockage occurrence, mass flow rates of unblocked microdevices are significantly increased under the total flow control (Fig. 4 left), while they are kept constant at the value in a normal condition under pressure drop control (Fig. 4 right). These results show that the proposed pressure drop control is effective in making flow distribution uniform even when blockage occurs.

\section{Comparison of control structures in pressure drop control}

In the previous section, it was confirmed that pressure drop control is superior to total flow control in realizing uniform flow distribution among unblocked microdevices when blockage occurs. In this section, two different control structures based on pressure drop 
control, pumping pressure control and pressure drop control over the parallelized section are investigated.

\section{3-1. Experimental apparatus}

Micro chemical plants having four or eight parallelized microdevices are used to grasp the distinction between two control structures. A schematic drawing of a micro chemical plant having four parallelized microdevices is shown in Fig. 5. Reactant is fed with a double plunger pump. The product line is open to the atmosphere. The flow rate of each microdevice is measured by using an in-line mass flow meter, and blockage in each microdevice is artificially realized by closing the valve located between the pump and each microdevice. Each microdevice consists of an SUS tube having $0.3 \mathrm{~mm}$ inner diameter and $2 \mathrm{~m}$ in length. In addition, an SUS tube with $0.5 \mathrm{~mm}$ in inner diameter and various lengths is installed after the parallelized microdevices to represent units that are not necessary to be parallelized. Hereafter, this section is referred to as a residence time section. The pressure drop over the parallelized section and the residence time section are denoted by $\triangle P a$ and $\triangle P b$, respectively. The ratio of $\triangle P a$ to $\triangle P b$ is changed by adjusting the length of the residence time section.

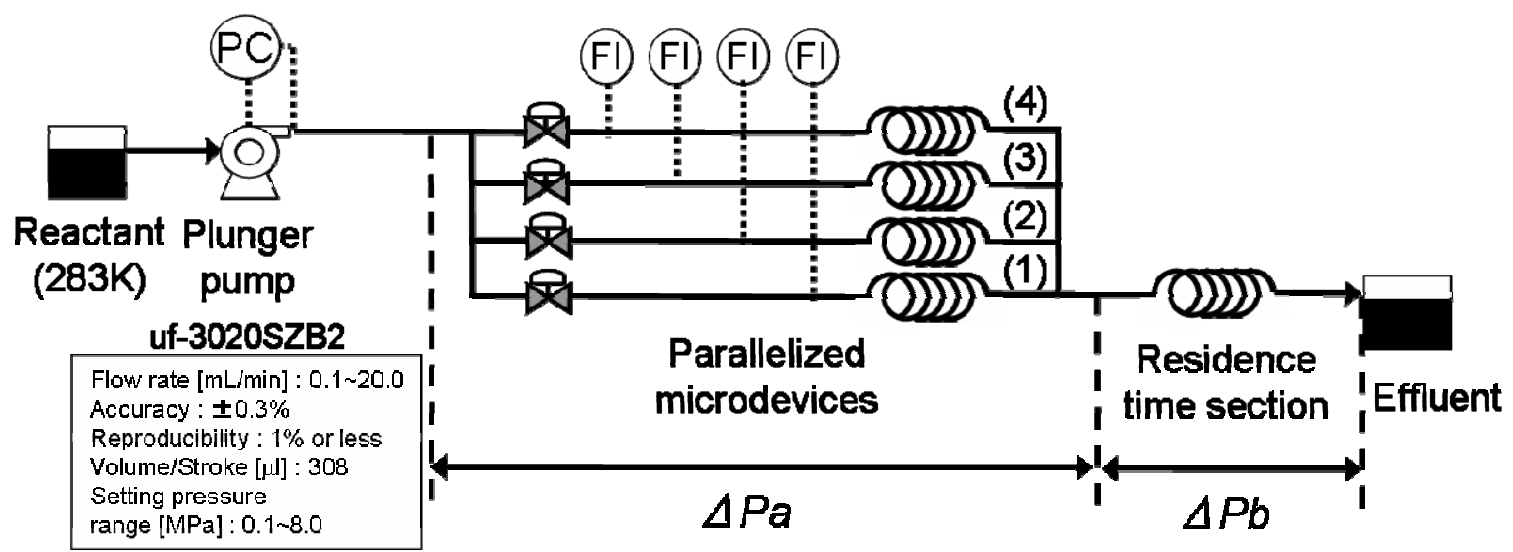

Fig. 5 Micro chemical plant under pumping pressure control 


\section{3-2. Pumping pressure control}

Under pumping pressure control, the double plunger pump is operated at constant pumping pressure. In experiments, pumping pressure is kept at a gauge pressure of 500 $\mathrm{kPa}$ to $1 \mathrm{MPa}$. Pressure drop over the whole micro chemical plant is kept constant under pumping pressure control, because the product line is open to the atmosphere.

The influence of blockage on flow distribution under pumping pressure control is investigated through both simulations and experiments with changing the ratio $\triangle P a / \triangle P b$ in the range of one-fifth to seven. The first step in the experimental procedure is to adjust the pumping pressure to realize a total flow rate of $12 \mathrm{~mL} / \mathrm{min}$. This operating condition is regarded as the normal condition. Then, microdevice 1 is artificially blocked by closing the valve. In 300 seconds, the micro chemical plant is returned to the normal condition by opening the valve. These procedures are repeated for the other valves to imitate blockage in the other microdevices.

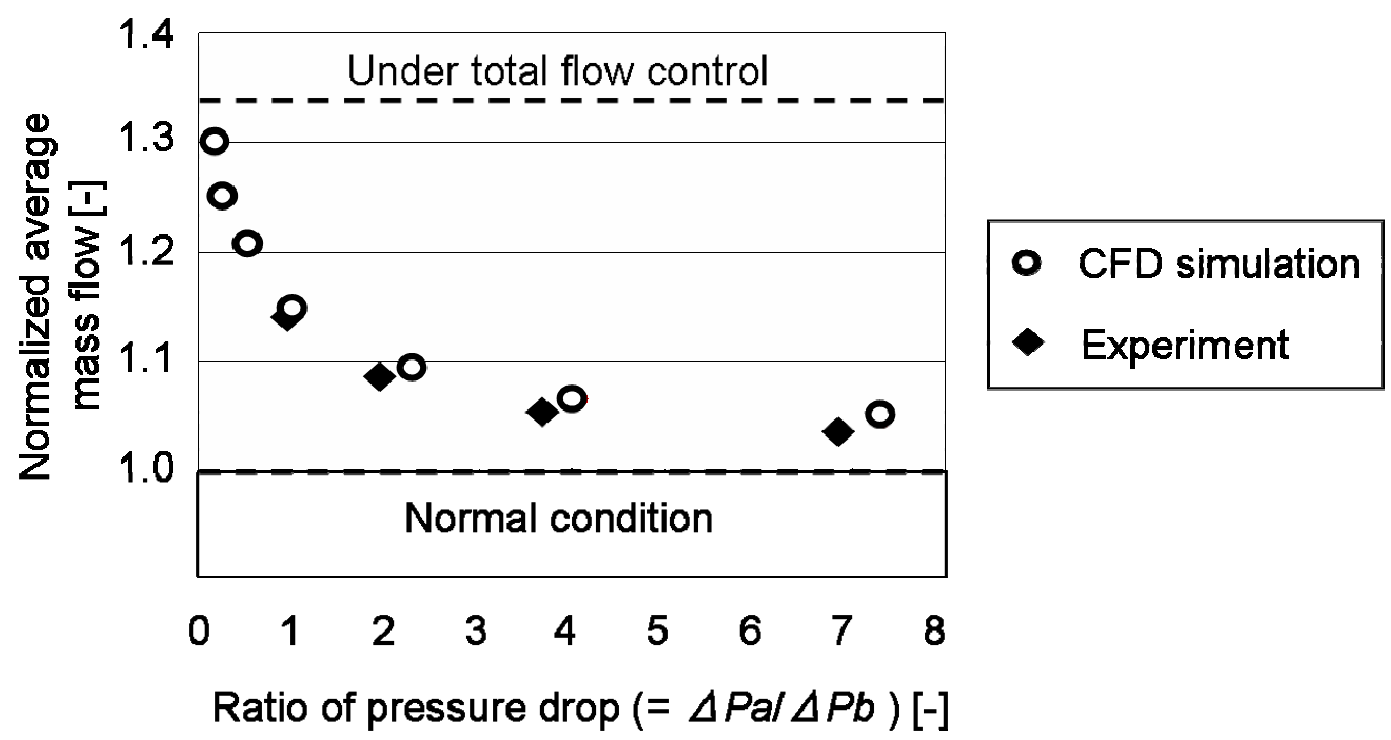

Fig. 6 Influence of blockage on the mass flow rate 
Figure 6 shows the normalized average mass flow rate, which is defined as the ratio of average mass flow rate of unblocked microdevices under blockage occurrence to that under the normal condition at each $\triangle P a / \triangle P b$. There is little difference between the results of experiments and those of CFD simulations. The normalized average mass flow rate becomes closer to the flow rate under the normal condition as $\triangle P a / \triangle P b$ becomes larger. In other words, $\triangle P a$ should be significantly larger than $\triangle P b$ to keep the flow rate of unblocked microdevices unchanged when blockage occurs.

In laminar flow, pressure drop is proportional to flow rate and flow resistance. Therefore, $\triangle P a$ and $\triangle P b$ are expressed by

$$
\begin{aligned}
& \Delta P a=\frac{1}{\sum_{i=1}^{4} \frac{1}{k_{i}}} F \\
& \Delta P b=k_{b} F
\end{aligned}
$$

where $k_{i}(i=1,2,3,4)$ is a coefficient of resistance in each microdevice, $k_{b}$ is a coefficient of resistance in the residence time section, and $F$ is the total flow rate. The pressure drop over the entire chemical plant, $\Delta P$, is calculated by $\Delta P=\Delta P a+\Delta P b=\left(\frac{1}{\sum_{i=1}^{4} \frac{1}{k_{i}}}+k_{b}\right) F$

When $\triangle P a$ is much larger than $\triangle P b$, the second term on the right side of Eq. (3) can be neglected. When blockage occurs in the $i$ th microdevice, $k_{i}$ becomes infinite. Consequently, the total flow rate under blockage occurrence becomes three-fourth times as much as that under the normal condition, provided that all the coefficients of flow resistance in the parallelized section are the same. Therefore, the flow rates of unblocked microdevices do not change when $\triangle P a / \triangle P b$ is sufficiently large. On the other hand, when 
$\triangle P a / \triangle P b$ is equal to 1 , the total flow rate under blockage occurrence becomes six-seventh times, and the flow rate of each unblocked microdevice under blockage occurrence becomes eight-seventh times as much as that under the normal condition. These results correspond to the results of CFD simulations and experiments. It is concluded that pumping pressure control is effective to realize uniform flow distribution when the pressure drop over the parallelized section is dominant.

\section{3-3. Pressure drop control over the parallelized section}

The flow uniformity achieved by pumping pressure control depends on $\triangle P a / \triangle P b$, which is the ratio of the pressure drop over the parallelized section to that over the residence time section. The flow uniformity in the parallelized microdevices deteriorates when $\triangle P a / \triangle P b$ is small. In this subsection, another pressure drop control structure where $\triangle P a$ is directly controlled by manipulating the flow rate of the bypass line is proposed.

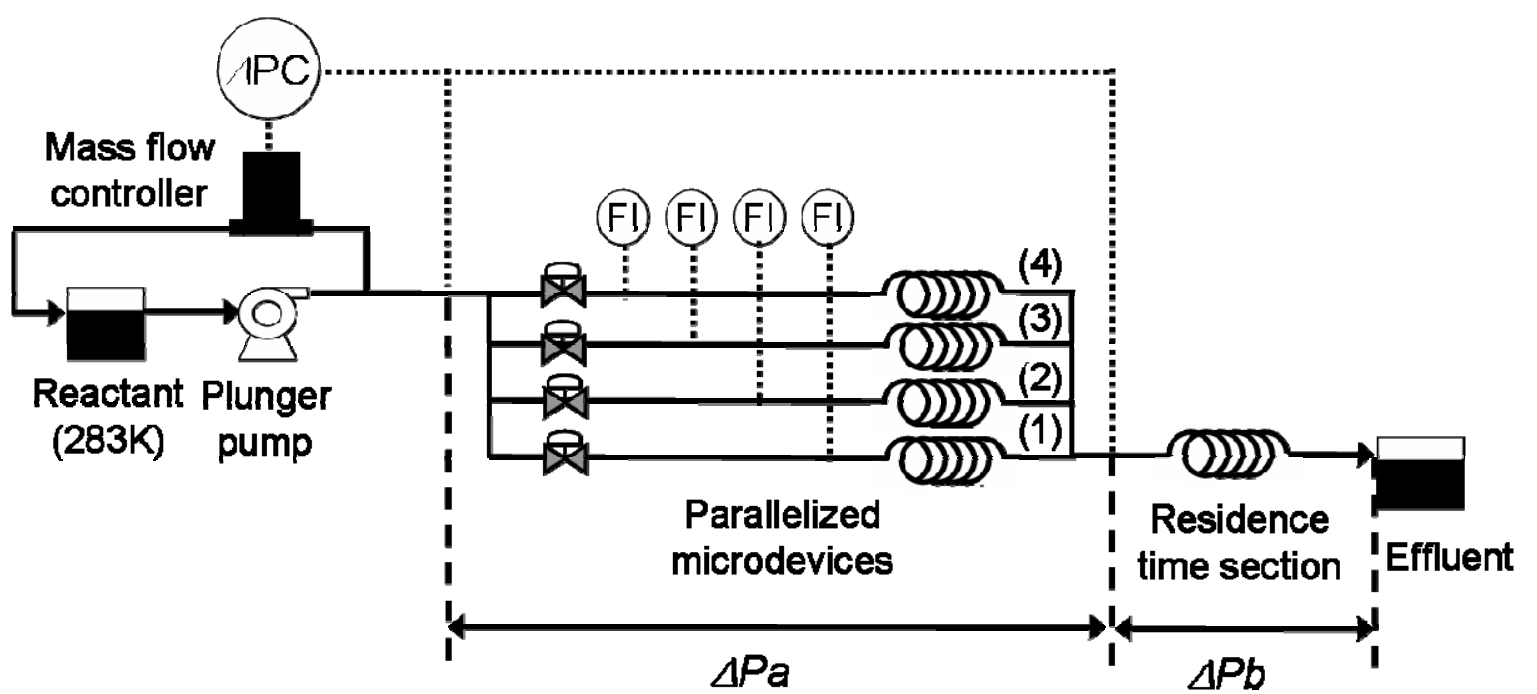

Fig. 7 Micro chemical plant under pressure drop control over the parallelized section

Table 1 Experimental conditions

\begin{tabular}{|c|c|c|c|c|c|c|c|}
\hline \multirow[b]{2}{*}{$\begin{array}{l}\text { Flow rate of the double } \\
\text { plunger pump [mL/min] }\end{array}$} & \multirow[b]{2}{*}{$\begin{array}{l}\text { Pressure drop over the } \\
\text { parallelized section }[\mathrm{kPa}]\end{array}$} & \multicolumn{3}{|c|}{ PID controller } & \multicolumn{3}{|c|}{ Residence time section } \\
\hline & & $\begin{array}{l}\text { Proportional } \\
\text { band [\%] }\end{array}$ & $\begin{array}{l}\text { Integral } \\
\text { time [s] }\end{array}$ & $\begin{array}{c}\text { Derivative } \\
\text { time [s] }\end{array}$ & $\begin{array}{c}\text { Inner } \\
\text { diameter [mm] }\end{array}$ & $\begin{array}{l}\text { Length } \\
\text { (Type A) [m] }\end{array}$ & $\begin{array}{c}\text { Length } \\
\text { (Type B) }[\mathrm{m}]\end{array}$ \\
\hline 18 & 500 & 34.6 & 7 & 1 & 0.5 & 0.1 & 4 \\
\hline
\end{tabular}




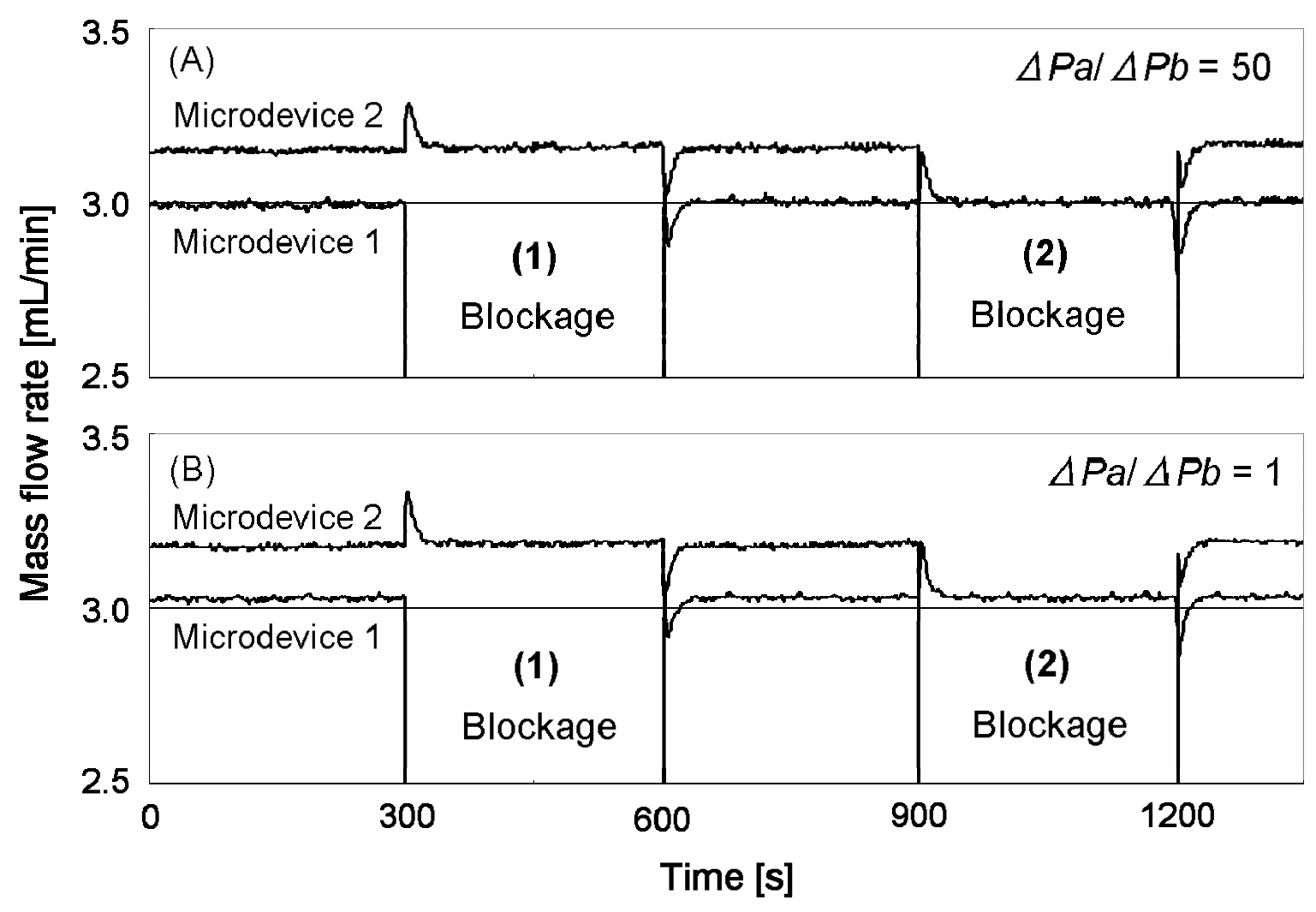

Fig. 8 The time series of mass flow rate in each microdevice in Type A: (A) $\triangle P a / \triangle P b=$ 50, (B) $\Delta P a / \triangle P b=1$

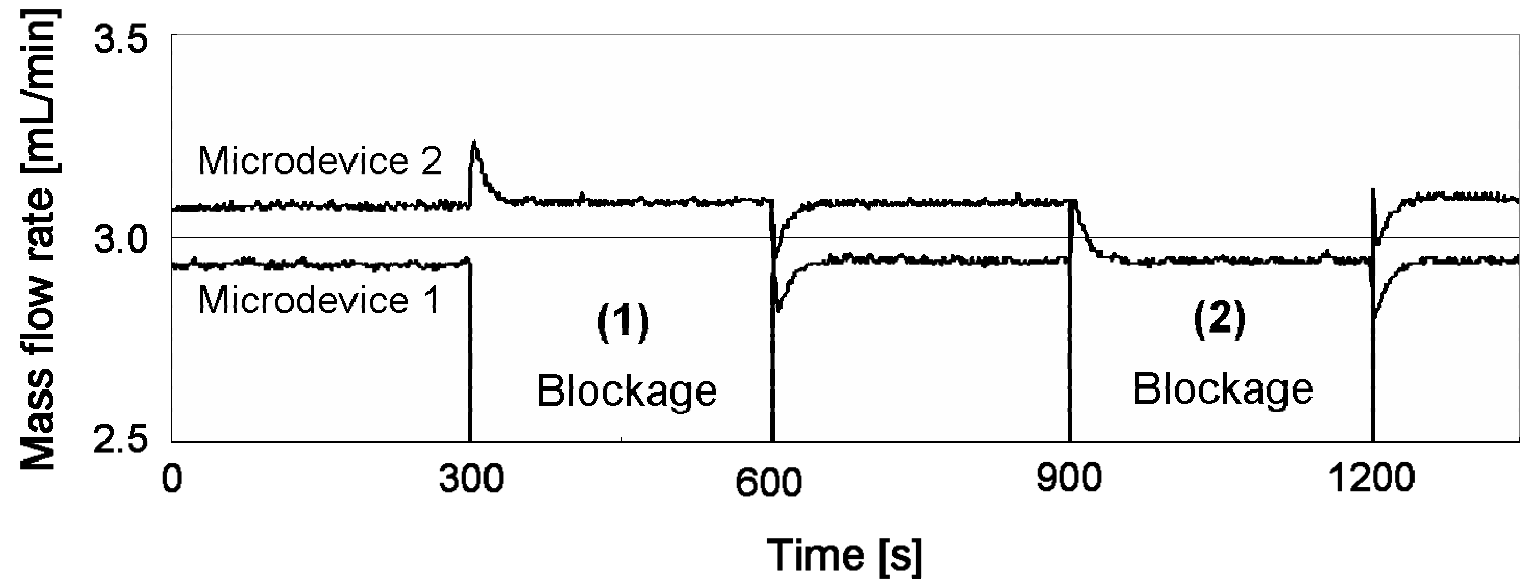

Fig. 9 The time series of mass flow rate in each microdevice in Type B 
The performance of the proposed control structure is evaluated experimentally by using the micro chemical plants with four (Type A) and eight (Type B) parallelized microdevices. Figure 7 shows an experimental setup of a Type A plant. A bypass line is equipped around the plunger pump. A mass flow controller, which is a PID controller, is installed in the bypass line to keep the pressure drop over the parallelized section $(\triangle P a)$ constant at its set-point. The experimental conditions are summarized in Table 1. The plunger pump is operated so that the total flow rate is kept constant. The other conditions are the same as those in the previous subsection.

The experimental results for a Type A plant are shown in Fig. 8. The top and bottom figures correspond to the case where $\triangle P a / \triangle P b=50$ and $\triangle P a / \triangle P b=1$, respectively. In the range of 0 to 300 seconds, the micro chemical plant is operated under the normal condition. The difference in flow rate between microdevices 1 and 2 is due to the degree of precision in the fabrication. When blockage occurs in microdevice 1 at 300 seconds, the flow rate of microdevice 1 goes to zero instantaneously and the flow rate of microdevice 2 deviates from its set-point. However, the flow rate of microdevice 2 returns to the normal level in a few tens of seconds. Flow rate deviation becomes small when blockage occurs gradually. The transient responses of microdevices 3 and 4 are similar to that of microdevice 2; they are not shown in Fig. 8 to identify the transient response of each microdevice easier. The top and bottom figures in Fig. 8 show almost the same profiles. This result shows that the efficiency of the proposed control structure does not depend on $\triangle P a / \triangle P b$.

The experimental result of a Type B plant is shown in Fig. 9, and it is almost the same as that of the Type A plant. These results show that the proposed control structure has the function of keeping the flow rate of the unblocked devices constant regardless of the changes in $\triangle P a / \triangle P b$ and the number of parallelized microdevices. 


\section{Application to parallelized micro heat exchangers}

Pressure drop control over the parallelized section is applied to a micro chemical plant having four parallelized micro heat exchangers.

\section{4-1. Experimental setup}

Figure 10 shows a schematic drawing of an extended micro chemical plant. The parallelized section in Fig. 7 is modified to have the function of heat exchange. A cocurrent type double-tube heat exchanger, which is made of stainless steel, is installed in each line. The inner tube has an inner diameter of $0.776 \mathrm{~mm}$ and outer diameter of 1.59 $\mathrm{mm}$. The outer tube has an inner diameter of $1.78 \mathrm{~mm}$ and outer diameter of $3.18 \mathrm{~mm}$. The length of the double-tube is $0.15 \mathrm{~m}$. The inlet and outlet temperatures and flow rate of fluid in each micro heat exchanger are monitored with thermocouples and an in-line mass flow meter, respectively.

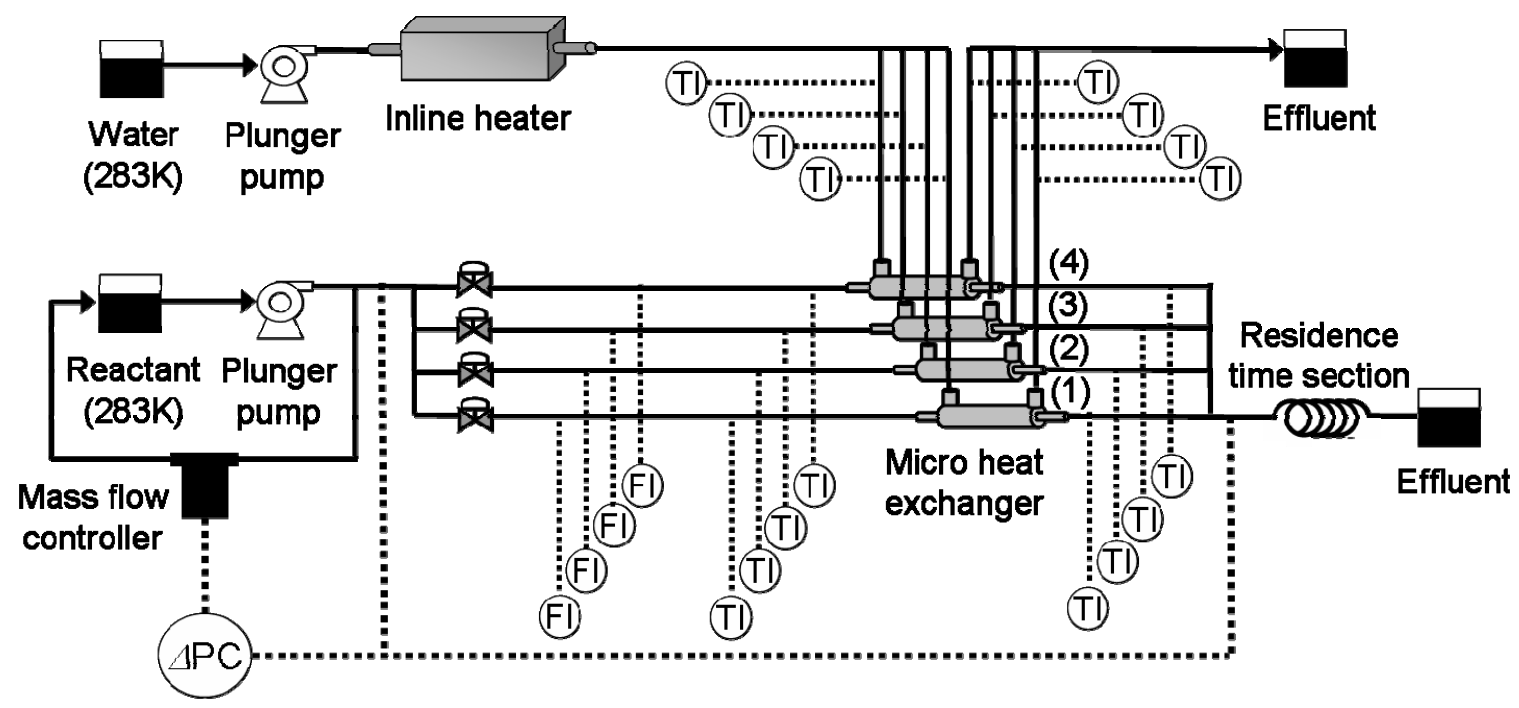

Fig. 10 Micro chemical plant having parallelized micro heat exchangers 
A plunger pump feeds a reactant at constant flow rate of $20 \mathrm{~mL} / \mathrm{min}$. Reactant is fed to the inner tubes of the double-tube micro heat exchangers. The outlet temperature of reactant is specified at $323 \mathrm{~K}$. On the other hand, water is used as a heat resource and heated from $283 \mathrm{~K}$ to $333 \mathrm{~K}$ by using an in-line heater. Heated water is fed to the outer tubes of the double-tube micro heat exchangers at a constant flow rate of $60 \mathrm{~mL} / \mathrm{min}$.

Under the above conditions, the overall heat transfer coefficient is calculated to be about $857 \mathrm{~J} /\left(\mathrm{m}^{2} \cdot \mathrm{s} \cdot \mathrm{K}\right)$ from Eqs. (4) to (6).

$$
\begin{aligned}
& Q=W_{c} \hat{C}_{p c}\left(T_{c 2}-T_{c 1}\right) \\
& Q=W_{h} \hat{C}_{p h}\left(T_{h 1}-T_{h 2}\right) \\
& Q=U A \frac{\left(T_{h 2}-T_{c 2}\right)+\left(T_{h 1}-T_{c 1}\right)}{2}
\end{aligned}
$$

where $Q[\mathrm{~J} / \mathrm{s}]$ is heat transfer rate, $U\left[\mathrm{~J} /\left(\mathrm{m}^{2} \cdot \mathrm{s} \cdot \mathrm{K}\right)\right]$ is overall heat transfer coefficient, $A$

$\left[\mathrm{m}^{2}\right]$ is heat transfer area, $T[\mathrm{~K}]$ is temperature of fluid, $W[\mathrm{~kg} / \mathrm{s}]$ is mass flow rate and $\hat{C}_{p}$ $[\mathrm{J} /(\mathrm{kg} \cdot \mathrm{K})]$ is specific heat capacity. The subscripts $c, h, 1$ and 2 denote reactant, water, inlet and outlet, respectively. In this work, the effect of blockage on the heat transfer rate in each line is investigated experimentally, and the results are discussed in the following subsection.

\section{4-2. Experimental results}

The time series of heat transfer rates in micro heat exchangers 1 and 2 are shown in Fig. 11. Heat transfer rates are calculated by Eq. (4). In the range of 0 to 300 seconds, the micro chemical plant is operated under the normal condition. The heat transfer rate in micro heat exchanger 2 is stable even when blockage occurs in micro heat exchanger 1 , which confirms the effectiveness of the proposed control structure. However, the heat transfer rate in micro heat exchanger 1 cannot instantaneously get back to the normal level 
when micro heat exchanger 1 is returned to the normal condition by opening the valve at 600 seconds. This phenomenon is caused by heat radiation from the pipe. The thermocouple is set up a little apart from the outlet of the micro heat exchanger as shown in Fig. 12. When blockage occurs in micro heat exchanger 1 and the reactant stops flowing, the pipe after the micro heat exchanger is rapidly cooled by thermal radiation. When the micro heat exchanger is recovered from blockage and the reactant starts to flow again, the heat transfer rate in micro heat exchanger 1 jumps to about $0.5 \mathrm{~kJ} / \mathrm{s}$ because the flow rate changes step-wise from zero to the normal level. However, the heat transfer rate does not get back to the normal level instantaneously because the enthalpy of the reactant is consumed to increase the temperature of the pipe and the reactant temperature rises gradually. The speed of the temperature change depends on the heat capacity of the pipe. The heat capacity of microdevices significantly affects the process dynamics, while the heat capacity of conventional macro equipment does not. To improve the heat exchange performance after the micro heat exchanger is recovered from blockage, a countermeasure against heat radiation is necessary.

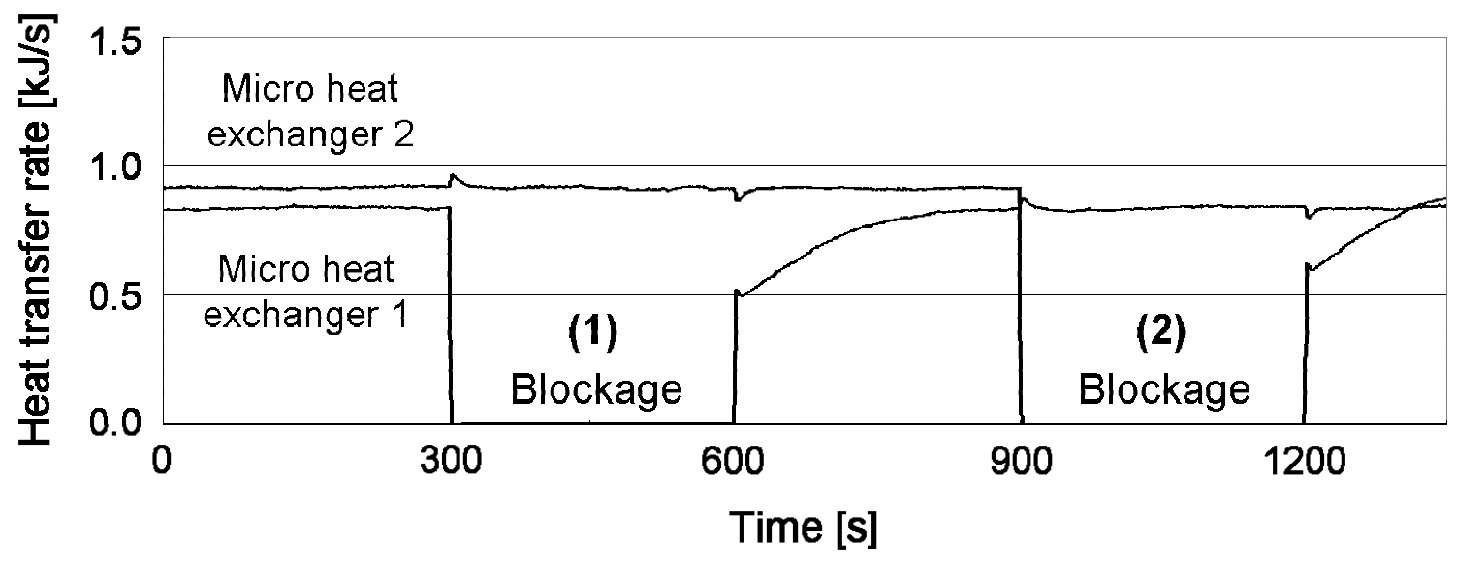

Fig. 11 The time series of heat transfer rate under pressure drop control over the parallelized section 


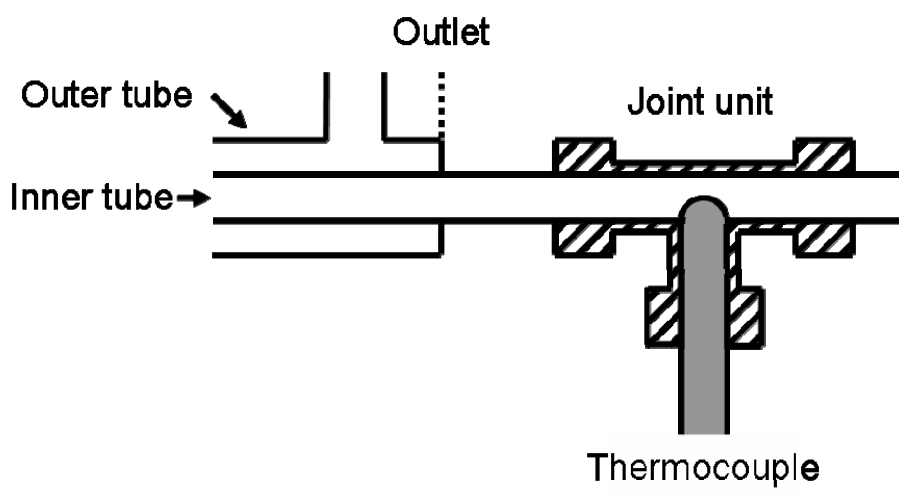

Fig. 12 The location of a thermocouple

The heat transfer rate in Fig. 11 represents the characteristics of not only the micro heat exchanger itself but also the pipe connected to the heat exchanger, because the thermocouples are set up a little apart from the micro heat exchanger. To evaluate the performance of the micro heat exchanger with accuracy, the thermocouples need to be installed closer to it. In general, the influence of sensing devices on fluids needs to be reduced, and sensing devices suitable for microdevices need to be developed for industrializing micro chemical plants.

\section{Blockage occurrence in three microdevices}

Provided that more than one microdevice is blocked at the same time, the effectiveness of the proposed pressure drop control over the parallelized section is investigated by using the micro chemical plant shown in Fig. 7. Figure 13 represents the time series of flow rates of microdevices 1 and 2. In the range of 150 to 480 seconds, it is apparent that the flow rate of microdevice 1 violently oscillates when microdevices 2,3 , and 4 are blocked simultaneously. 


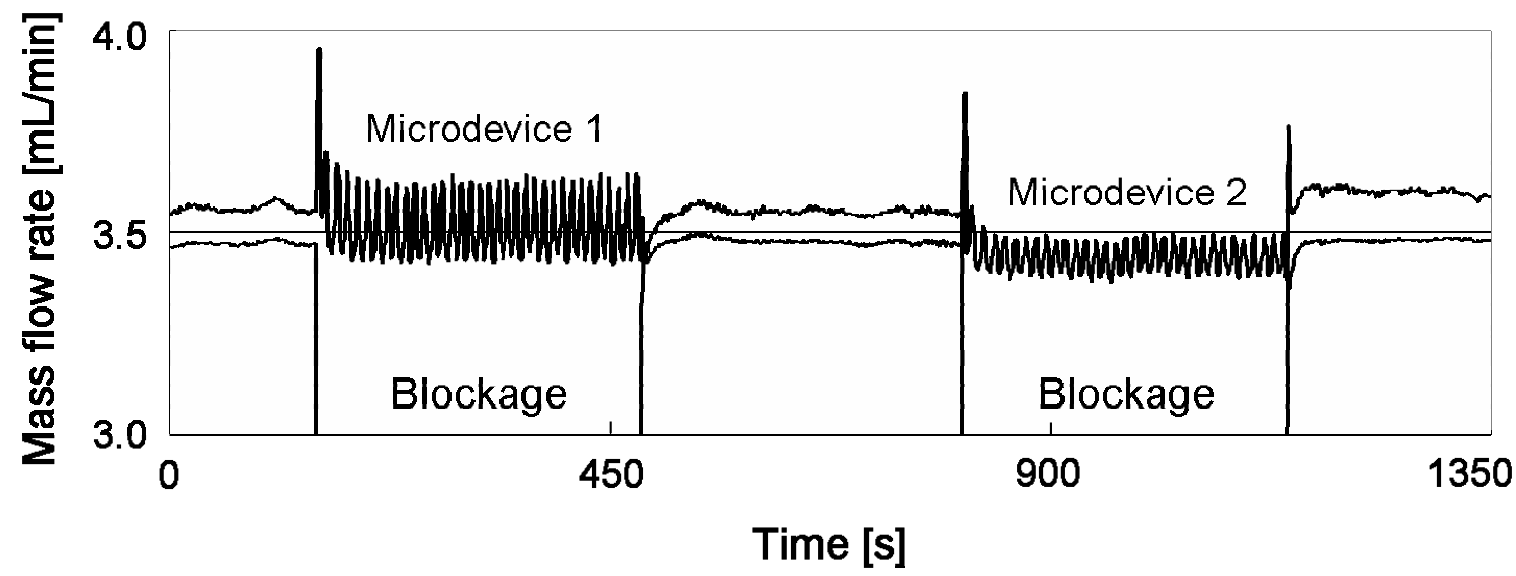

Fig. 13 The time series of mass flow rate under blockage occurrence in three microdevices

This result is explained as follows: the feed flow rate $F_{\text {Total }}$ of the plunger pump under the normal condition is expressed by

$$
F_{\text {Total }}=4 F_{M D}+F_{\text {Bypass }}
$$

where $F_{M D}$ and $F_{\text {Bypass }}$ denote the flow rate in each microdevice and that in the bypass line, respectively. $F_{\text {Bypass }}$ is assumed to be perfectly controlled by the mass flow controller. When one microdevice is blocked (Case 1), the flow rate of the bypass line $\hat{F}_{\text {Bypass }}$ is represented by

$$
\hat{F}_{\text {Bypass }}=F_{\text {Total }}-3 F_{M D}
$$

At this time, $F_{\text {Total }}$ and $F_{M D}$ in Case 1 are the same as those under the normal condition. On the other hand, when the three microdevices are blocked (Case 2), the flow rate of the bypass line $\hat{\hat{F}}_{\text {Bypass }}$ is represented by

$$
\hat{\hat{F}}_{\text {Bypass }}=F_{\text {Total }}-F_{M D}
$$

Under these conditions, when the flow rate in the bypass line changes slightly, Eqs (8) and (9) are expressed by 


$$
\begin{aligned}
& F_{\text {Total }}=3\left(F_{M D}+\delta \hat{F}_{M D}\right)+\hat{F}_{\text {Bypass }}-\delta F_{\text {Bypasss }} \\
& F_{\text {Total }}=\left(F_{M D}+\delta \hat{\hat{F}}_{M D}\right)+\hat{\hat{F}}_{\text {Bypass }}-\delta F_{\text {Bypasss }}
\end{aligned}
$$

According to Eqs. (10) and (11), the relationship between the change of the flow rate of the bypass line and that of each microdevice is expressed by

$$
\begin{gathered}
\delta \widetilde{F}_{M D}=\frac{\delta F_{\text {Bypasss }}}{3} \\
\delta \widetilde{\widetilde{F}}_{M D}=\delta F_{\text {Bypasss }}
\end{gathered}
$$

Judging from Eqs. (12) and (13), the effect of $\delta F_{\text {Bypasss }}$ on the flow rate of each microdevice in Case 2 becomes three times as much as that in Case1. Since $F_{M D}$ is proportional to $\triangle P a$, the steady-state gain of the process in Case 2 is three times larger than that in Case 1. This change in the steady-state gain affects the control performance significantly and causes oscillatory behavior in Case 2, as shown in Fig. 13. In general, the control action becomes stronger and the controlled and manipulated variables become more oscillatory as the number of blocked microdevices increases, as long as the same controller is used.

The influence of blockage on the control performance depends greatly on the ratio of the number of blocked microdevices to the number of parallelized microdevices. As the ratio is smaller, the influence is smaller. The influence will be significant when there are a few parallelized microdevices, as shown in this experimental study. In such a situation, it is necessary to design a controller that is robust against the degree of blockage. For example, control parameters might need to be changed appropriately according to the number of blocked microdevices. 


\section{Conclusion}

We have discussed operation policies and control structures for micro chemical plants with an external numbering-up structure. Micro chemical plants having four or eight parallelized microdevices and a residence time section were constructed, and the flow distribution among the parallelized microdevices was investigated when one or more microdevice was completely blocked.

Two operation policies, total flow control and pressure drop control, were compared. The simulation result shows that the pressure drop control is effective to keep a uniform flow distribution among the parallelized microdevices even when blockage occurs. In addition, two control structures based on pressure drop control, pumping pressure control and pressure drop control over the parallelized section, were investigated experimentally. The former control structure is simple. However, this structure functions only when the ratio of the pressure drop over the parallelized section to that over the residence time section, $\triangle P a / \triangle P b$, is large. On the other hand, the latter control structure can make the flow distribution uniform for any $\triangle P a / \triangle P b$, and its effectiveness was confirmed through a further experiment with a micro chemical plant having parallelized micro heat exchangers.

The investigations mentioned above were conducted on the assumption that a microdevice is completely blocked and its flow rate becomes zero. In an actual process, there is a possibility that microdevices are partially blocked. In such a case, uniformity of the residence time in the parallelized microdevices cannot be achieved, and the flow maldistribution deteriorates reaction selectivity, heat transfer efficiency, and so on. When blockage in a microdevice is detected, it is preferable to stop production in the blocked microdevice and to replace it with a new microdevice. Meanwhile, the flow rates of the other unblocked microdevices are kept constant at the normal level by the pressure drop control over the parallelized section. 
The proposed pressure drop control over the parallelized section proves to be highly effective for the operation of micro chemical plants with external numbering-up structure. However, in addition to the control method, it is necessary to develop an effective monitoring method that can detect and diagnose abnormal conditions such as blockage. To realize stable operation of micro chemical plants over a long period of time, more active research on operation and monitoring should be conducted.

\section{Acknowledgment}

This research was partially supported by the New Energy and Industrial Technology Development Organization (NEDO), Project of Micro-Chemical Technology for Production, Analysis and Measurement Systems.

\section{References}

[1] DeWitt, H. S.; "Microreactors for chemical synthesis," Chemical Biology, 3, 350-356

[2] Hessel, V., and H. Loew; "Microchemical Engineering: Components, Plant Concepts, User Acceptance-Part III," Chem. Eng. Technol., 26(5), 531-544 (2003c)

[3] Fujioka, T., M. Kano, O. Tonomura, S. Hasebe; "Data-Based and Model-Based Blockage Diagnosis Systems for Stacked Micro Chemical Plants", AIChE Annual Meeting, paper 570b, Cincinnati, OH, Oct. 30-Nov. 4 (2005)

[4] Bayer, T., D. Pysall, and O. Wachesen; "Micro Mixing Effects in Continuous Radical Polymerization," Proceedings of the 3rd International Conference on Microreaction Technology (IMRET3), Frankfurt, Germany, 165-170 (1999) 